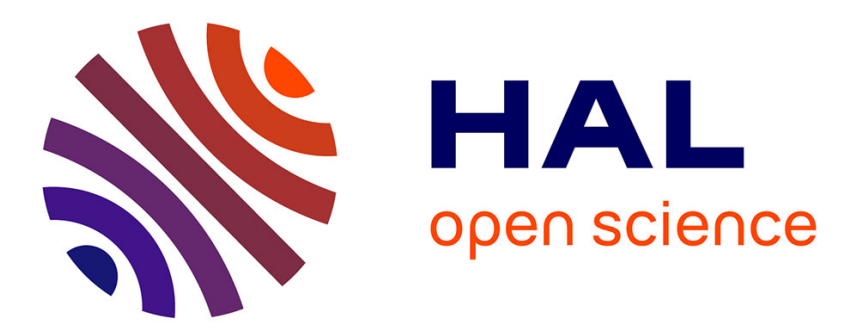

\title{
Extension of the natural element method to surface tension and wettability for the simulation of polymer flows at the micro and nano scales
}

Hubert Teyssèdre, Pierre Gilormini

\section{- To cite this version:}

Hubert Teyssèdre, Pierre Gilormini. Extension of the natural element method to surface tension and wettability for the simulation of polymer flows at the micro and nano scales. Journal of Non-Newtonian Fluid Mechanics, 2013, 200, pp.9-16. 10.1016/j.jnnfm.2012.10.003 . hal-00860109

\section{HAL Id: hal-00860109 https://hal.science/hal-00860109}

Submitted on 10 Sep 2013

HAL is a multi-disciplinary open access archive for the deposit and dissemination of scientific research documents, whether they are published or not. The documents may come from teaching and research institutions in France or abroad, or from public or private research centers.
L'archive ouverte pluridisciplinaire HAL, est destinée au dépôt et à la diffusion de documents scientifiques de niveau recherche, publiés ou non, émanant des établissements d'enseignement et de recherche français ou étrangers, des laboratoires publics ou privés. 


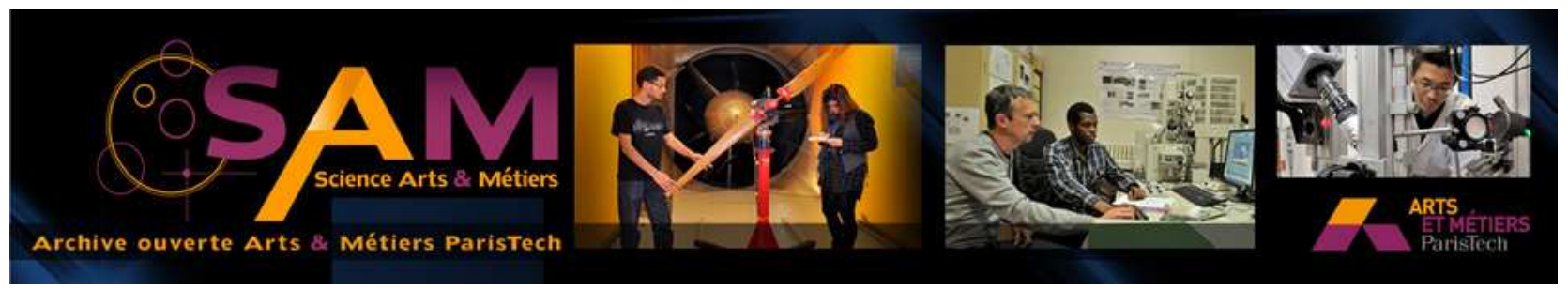

\section{Science Arts \& Métiers (SAM)}

is an open access repository that collects the work of Arts et Métiers ParisTech researchers and makes it freely available over the web where possible.

This is an author-deposited version published in: http://sam.ensam.eu

Handle ID: .http://hdl.handle.net/10985/7294

\section{To cite this version :}

Hubert TEYSSĖDRE, Pierre GILORMINI - Extension of the natural element method to surface tension and wettability for the simulation of polymer flows at the micro and nano scales - Journal of Non-Newtonian Fluid Mechanics - Vol. 200, p.9-16 - 2013 


\title{
Extension of the natural element method to surface tension and wettability for the simulation of polymer flows at the micro and nano scales
}

\author{
Hubert Teyssèdre ${ }^{\mathrm{a}, \mathrm{b}}$, Pierre Gilormini ${ }^{\mathrm{b}, *}$ \\ ${ }^{a}$ Silsef, 74160 Archamps, and CEA/LETI, 17 rue des Martyrs, 38054 Grenoble, France \\ ${ }^{b}$ PIMM, Arts et Métiers ParisTech, CNRS, 151 bvd de l'Hôpital, 75013 Paris, France
}

\begin{abstract}
The natural element method is used to simulate two-dimensional viscous flows where interfacial effects must be taken into account, for application to polymer melts at the micro and nano scales. The variational formulation includes surface tension on the free surfaces, a net wetting force is applied at the contact line where the fluid reaches a solid surface, and the Navier-slip condition is used along fluid-solid interfaces. No dynamic wetting angle is prescribed, and the contact angle obtained results from the other material parameters and from overall flow conditions. A comparison with an analytical solution in a simple surface tension-driven flow is given, and contact with a rigid solid is involved in the transient spreading of a droplet and in the steady movement of a meniscus between two plates.
\end{abstract}

Keywords: Polymers, Surface tension, Contact angle, Natural element method

\section{Introduction}

The flow of polymer melts at micro or nano scale involves very low Reynolds numbers and strong interfacial effects. This is especially true for thermal nanoimprint lithography [1], where nanometric patterns are engraved into a very thin film of polymer at high temperature, a process for which the simulation code described in this paper has been developed initially. The

\footnotetext{
*Corresponding author. Tel.: +33 144246337

Email address: pierre.gilormini@ensam.eu (Pierre Gilormini)
} 
relevant interfacial phenomena include surface tension on the free surfaces, wetting angle where a free surface reaches a solid, and slip between fluid and solids. The latter aspect is very common at small scales [2], but is difficult to characterize precisely [3].

Within the limits where continuum mechanics applies, this work uses the natural element method (NEM) to simulate such viscous flows. The principle of the NEM has been introduced by Traversoni [4], but the first application of this meshless method is due to Braun and Sambridge [5], who considered two-dimensional Stokes flows. The method has been applied later to solid mechanics by Sukumar et al. [6], whose work promoted the method and stimulated subsequent developments. A complete literature review of the NEM is beyond this paper, but the work of Cueto et al. [7] about non convex boundaries in solid mechanics and its extensions to the flows of complex fluids by Martínez et al. [8] and to fluid dynamics by González et al. [9] are worth noting in connection with the present work. Shallow water flows have also been simulated with the NEM by Ata et al. [10], who applied the technique developed by Yvonnet et al. [11] for non convex boundaries, and by Darbani et al. [12]. Moreover, surface tension has been included by Defauchy et al. [13] recently, for application to polymer powder sintering, but without fluid-solid contact. In contrast with the finite element method, the NEM employs natural neighbour interpolation without fixed connectivity and has low sensitivity to mesh distorsion. The NEM also has the advantage over such other meshless methods as the smooth particles hydrodynamics, to be a strictly interpolant method and thus to impose essential boundary conditions exactly (especially with the techniques of [7] or [11] on non convex boundaries). Moreover, the examples treated by González et al. [9] or Cueto et al. [14], among others, demonstrate the natural ability of the NEM to handle with free-surface flows, especially within a Lagrangian approach. These advantages make the NEM a promising method for the simulation of fluid flows.

The layout of this paper is as follows. First, the variational formulation used is derived after the governing equations have been recalled. This details how surface tension, wettability, and slip are taken into account in the two-dimensional flows considered. Then, three illustrative examples are discussed. A first application compares an analytical solution for a simple surface tension-driven flow, and the other two examples include fluid-solid contact in either a transient or a steady problem. 


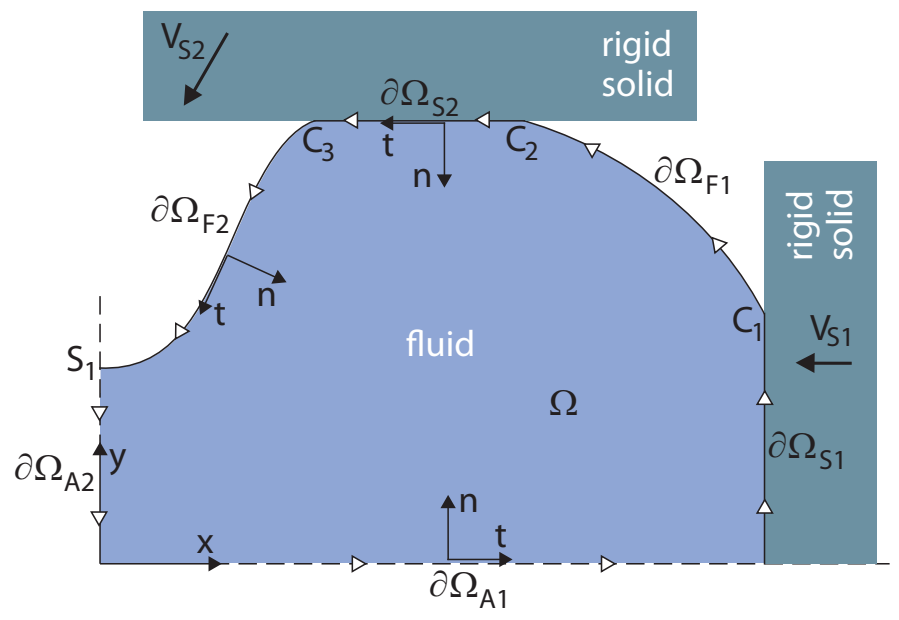

Figure 1: Notations and conventions used. White arrows show the (anticlockwise) orientation of the boundary of the fluid domain, which leads to the unit tangent and normal vectors. Symmetry axes are shown as broken lines.

\section{Variational formulation and numerical implementation}

The aim of this section is to detail the variational formulation that is applied in this study, using the notations and conventions shown in Fig. 1. The analysis is limited to two-dimensional flows with zero velocity component along the $z$ axis. Therefore, a section of the fluid volume and interacting solids in the $(x, y)$ plane is considered. The boundary $\partial \Omega$ of the fluid domain $\Omega$ consists of various parts that are either free surfaces $\left(\partial \Omega_{F}\right.$ set, possibly split into $\left.\partial \Omega_{F 1}, \partial \Omega_{F 2} \ldots\right)$, or located along symmetry axes $\left(\partial \Omega_{A}\right.$ set), or in contact with solids $\left(\partial \Omega_{S}\right.$ set). As shown in Fig. 1, the $\partial \Omega$ curve is oriented anticlockwise, which determines the orientation of the unit tangent vector $\boldsymbol{t}$, and the unit normal vector $\boldsymbol{n}$ is inwardly directed.

\subsection{Governing equations}

Inertial effects and gravity can be neglected in microflows and nanoflows of viscous liquids [15], since very low Reynolds numbers and small lengths compared to the capillary length are involved. In typical applications considered here, the length scale is of order $1 \mu \mathrm{m}$ or less, the velocities of order $1 \mu \mathrm{m} / \mathrm{s}$ or less, the viscosity of order $10^{4} \mathrm{~Pa} . \mathrm{s}$, and surface tension is about $40 \mathrm{mN} / \mathrm{m}$, which gives $R_{e}=10^{-13}$ and a capillary length of $2 \mathrm{~mm}$. Therefore, in the creeping incompressible flows of polymers considered, where all body 
forces are neglected, the equations to be satisfied in the fluid volume $\Omega$ by the velocity $\boldsymbol{v}$ and Cauchy stress tensor $\boldsymbol{\sigma}$ are merely

$$
\operatorname{div} \boldsymbol{v}=0 \quad \text { and } \quad \operatorname{div} \boldsymbol{\sigma}=\mathbf{0} .
$$

These two tensor fields are related by the constitutive equation

$$
\boldsymbol{\sigma}=2 \eta \boldsymbol{D}(\boldsymbol{v})-p \boldsymbol{I} \quad \text { with } \quad \boldsymbol{D}(\boldsymbol{v})=\frac{1}{2}\left[\operatorname{grad} \boldsymbol{v}+(\operatorname{grad} \boldsymbol{v})^{T}\right]
$$

where $\boldsymbol{D}$ is the strain rate tensor, $\boldsymbol{I}$ the second-order identity tensor and $p$ the hydrostatic pressure. Moreover, $\eta$ denotes the viscosity, which may be a function of the equivalent shear rate $\dot{\gamma}_{e q}=\sqrt{2 D: D}$ in the non-Newtonian shear-thinning case.

Along the fluid boundary $\partial \Omega$, different types of conditions apply on the $\partial \Omega_{A}, \partial \Omega_{F}$ and $\partial \Omega_{S}$ sets. Along symmetry axes, the no penetration condition writes

$$
\boldsymbol{v} \cdot \boldsymbol{n}=0 \quad \text { on } \quad \partial \Omega_{A}
$$

whereas Laplace's law gives

$$
\boldsymbol{t} . \boldsymbol{\sigma} \cdot \boldsymbol{n}=0 \quad \text { and } \quad \boldsymbol{n .} \cdot \boldsymbol{n} \boldsymbol{n}=-\gamma \kappa \quad \text { on } \quad \partial \Omega_{F}
$$

along the free surfaces, where $\gamma$ denotes the surface tension and $\kappa$ the curvature. With the conventions used, the latter is positive where the fluid boundary is convex, i.e. where the center of curvature lies on the fluid side (in the direction of $\boldsymbol{n}$ ). At such points as $C_{1}, C_{2}$ and $C_{3}$ in Fig. 1 or at point $C$ in Fig. 2, where the free boundary of the polymer reaches the surface of a solid, the tangent vector is discontinuous, which defines a contact angle $\theta$, and we apply a concentrated net wetting force, parallel to the tangent $\boldsymbol{t}_{S}$ to the solid-fluid interface,

$$
\boldsymbol{f}_{w}=\epsilon \gamma\left(\boldsymbol{t}_{F} \cdot \boldsymbol{t}_{S}+\cos \theta_{s}\right) \boldsymbol{t}_{S} \quad \text { at } \quad \partial \Omega_{F} \cap \partial \Omega_{S}
$$

where $\theta_{s}$ denotes the static wetting (equilibrium) angle. In the above definition, $\epsilon=1$ if vector $\boldsymbol{t}_{F}$ (tangent to the free surface) points inside $\partial \Omega_{F}$ (at $C_{1}$ and $C_{3}$ in Fig. 1 or at $C$ in Fig. 2, for instance), and $\epsilon=-1$ otherwise (at $C_{2}$ in Fig. 1, for instance). This force per unit length of contact line is zero when $\boldsymbol{t}_{F} \cdot \boldsymbol{t}_{S}=-\cos \theta_{s}$ (the contact angle is equal to the static wetting angle), otherwise $\boldsymbol{f}_{w}$ tends to move the contact point so that the contact angle gets closer to the static wetting angle. This simple way of introducing 


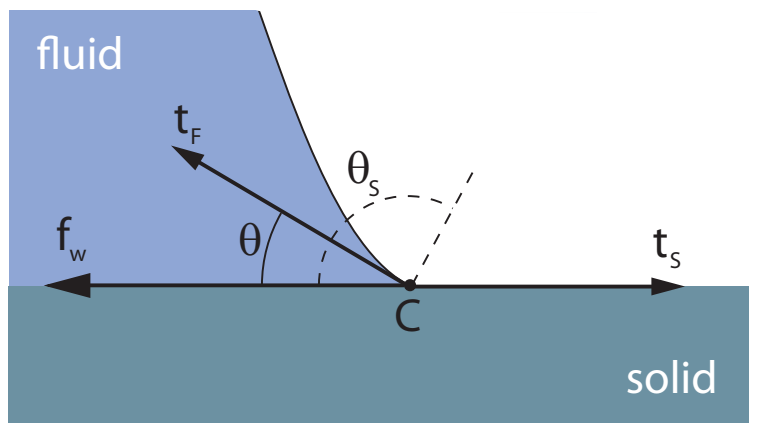

Figure 2: Tangent net wetting force at a contact point.

wettability does not enforce the contact angle value and allows for a difference between advancing and receding fronts, since the contact angle will be influenced by the whole flow field. Expression (5) is obtained immediately (as in De Gennes et al. [16], for instance) by assuming that the three surface tensions involved in Young's equation are maintained in dynamic cases, but out of balance. Therefore, neither the more elaborate model of [17] with dynamic surface tension nor the complex expression of the net wetting force derived in [18] is considered here. This also departs from the approach of $\breve{S i k a l o ~ e t ~ a l . ~[19] ~ f o r ~ v o l u m e-o f-f l u i d ~ s i m u l a t i o n s, ~ w h e r e ~ t h e ~ f o r c e ~ u s e d ~ m i s s e s ~}$ the first term in (5). Here, as expected, there is no singularity in static equilibrated configurations of the fluid and the non-zero net wetting force induces a singularity in dynamic situations where the contact angle differs from the static angle. It may be mentioned finally that Young's law also leads to a force component at the contact line that is normal to the solid surface, with an amplitude $\gamma \sin \theta$ per unit length. This component, which is not zero but $\gamma \sin \theta_{s}$ at equilibrium, will not be considered further since the solid has a prescribed velocity in the problems considered here, but it should be taken into account if the solid were free to move.

As is well known in the literature about the moving contact line problem (see Shikhmurzaev [17] for an extensive review), the standard no-slip boundary condition at fluid-solid interfaces leads to a non-integrable force singularity at contact points [20], and a standard procedure to make it integrable consists in using a slip condition instead [21]. At all points where the fluid domain contacts a rigid solid, a Navier-slip boundary condition is considered in the present work, which writes

$$
\boldsymbol{n} .\left(\boldsymbol{v}-\boldsymbol{v}_{S}\right)=0 \quad \text { and } \quad \boldsymbol{t} .\left(\boldsymbol{v}-\boldsymbol{v}_{S}\right)=\beta \boldsymbol{t} . \boldsymbol{\sigma} . \boldsymbol{n} \quad \text { on } \quad \partial \Omega_{S}
$$


where $\boldsymbol{v}_{S}$ denotes the velocity of the solid. The first equation results from the no penetration condition and the second one introduces the slip coefficient $\beta$. If the fluid is Newtonian, with a fixed viscosity $\eta$, selecting a $\beta$ value is equivalent to choosing a slip length $b=\beta \eta$, but this is not true for non-Newtonian shear-thinning fluids, since $\eta$ varies. Of course, the no-slip condition is recovered when $\beta=0$, and frictionless slip is obtained when $\beta \rightarrow \infty$.

\subsection{Weak form}

Combining the first relation in (2) and the second equation in (1) to eliminate $\boldsymbol{\sigma}$, multiplying by a test velocity field $\boldsymbol{v}^{\star}$ and integrating over the fluid domain leads to

$$
\forall \boldsymbol{v}^{\star}, \quad 2 \int_{\Omega} \boldsymbol{v}^{\star} \cdot \operatorname{div}(\eta \boldsymbol{D}(\boldsymbol{v})) \mathrm{d} S-\int_{\Omega} \boldsymbol{v}^{\star} \cdot \operatorname{grad} p \mathrm{~d} S=0
$$

where $\mathrm{d} S$ denotes the surface element of the fluid section $\Omega$. Similarly, multiplying the first equation in (1) by a test pressure field $p^{\star}$ and integrating over the fluid region gives

$$
\forall p^{\star}, \quad \int_{\Omega} p^{\star} \operatorname{div} \boldsymbol{v} \mathrm{d} S=0
$$

The $\boldsymbol{v}^{\star}$ field is continuous with piecewise continuous derivatives and satisfies

$$
\boldsymbol{v}^{\star} \cdot \boldsymbol{n}=0 \quad \text { on } \quad \partial \Omega_{A} \quad \text { and } \quad \partial \Omega_{S}
$$

whereas the $p^{\star}$ field is piecewise continuous. Integration of (7) by parts and combination with (2) gives

$$
\begin{aligned}
\forall \boldsymbol{v}^{\star}, \quad 2 \int_{\Omega} \eta \boldsymbol{D}\left(\boldsymbol{v}^{\star}\right): \boldsymbol{D}(\boldsymbol{v}) \mathrm{d} S & -\int_{\Omega} p \operatorname{div} \boldsymbol{v}^{\star} \mathrm{d} S- \\
& -\sum_{C_{i}} \boldsymbol{v}^{\star} \cdot \boldsymbol{f}_{w}+\int_{\partial \Omega} \boldsymbol{v}^{\star} \cdot \boldsymbol{\sigma}(\boldsymbol{v}, p) \cdot \boldsymbol{n} \mathrm{d} l=0
\end{aligned}
$$

where $\mathrm{d} l$ denotes the element of boundary $\partial \Omega$ of the fluid section and where the contribution of concentrated net wetting forces at contact points has been included duly. There remains now to use the boundary conditions to compute the last term, where $\partial \Omega=\partial \Omega_{A} \cup \partial \Omega_{F} \cup \partial \Omega_{S}$. 
Since $\boldsymbol{\sigma} \cdot \boldsymbol{n}$ is parallel to $\boldsymbol{n}$ along a symmetry axis, (9) leads to a zero contribution of the last term of (10) over $\partial \Omega_{A}$. Using (4), the contribution over $\partial \Omega_{F}$ writes

$$
\int_{\partial \Omega_{F}} \boldsymbol{v}^{\star} \cdot \boldsymbol{\sigma} \cdot \boldsymbol{n} \mathrm{d} l=-\int_{\partial \Omega_{F}} \gamma \kappa \boldsymbol{v}^{\star} \cdot \boldsymbol{n} \mathrm{d} l .
$$

The restriction of the present work to plane flows allows using the procedure introduced by Ruschak [22], which takes advantage of the special expression of curvature in that case

$$
\kappa \boldsymbol{n}=\frac{\mathrm{d} \boldsymbol{t}}{\mathrm{d} l}
$$

and leads to

$$
\begin{aligned}
& \int_{\partial \Omega_{F}} \boldsymbol{v}^{\star} \cdot \boldsymbol{\sigma} \cdot \boldsymbol{n} \mathrm{d} l=-\int_{\partial \Omega_{F}} \gamma \boldsymbol{v}^{\star} \cdot \mathrm{d} \boldsymbol{t}= \\
& \quad=\int_{\partial \Omega_{F}} \gamma \boldsymbol{t} \cdot\left(\operatorname{grad} \boldsymbol{v}^{\star}\right) \cdot \boldsymbol{t} \mathrm{d} l+\sum_{S_{i}, C_{i}} \epsilon \gamma \boldsymbol{v}^{\star} \cdot \boldsymbol{t}_{F}
\end{aligned}
$$

after integration by parts. The above finite sum over points $S_{i}$ and $C_{i}$ where the free surface of the fluid reaches a symmetry axis or a rigid solid, respectively, uses $\epsilon$ as already defined above (with $\epsilon=-1$ at $S_{1}$ in Fig. 1, for instance). The above finite sum may be limited to contact points $C_{i}$, since (9) nullifies the contribution of points $S_{i}$. Moreover, (9) gives also

$$
\boldsymbol{v}^{\star} \cdot \boldsymbol{t}_{F}=\left(\boldsymbol{v}^{\star} \cdot \boldsymbol{t}_{S}\right) \boldsymbol{t}_{S} \cdot \boldsymbol{t}_{F}
$$

at points $C_{i}$.

Finally, the Navier slip condition (6) can be used to compute the contribution of $\partial \Omega_{S}$ to the last term of (10) as

$$
\int_{\partial \Omega_{S}} \boldsymbol{v}^{\star} \cdot \boldsymbol{\sigma} \cdot \boldsymbol{n} \mathrm{d} l=\int_{\partial \Omega_{S}} \frac{1}{\beta} \boldsymbol{v}^{\star} \cdot\left(\boldsymbol{v}-\boldsymbol{v}_{S}\right) \mathrm{d} l
$$

since $\boldsymbol{v}^{\star}$ is parallel to $\boldsymbol{t}$ on $\partial \Omega_{S}$ because of (9) and, therefore, (15) is equivalent to the second relation in $(6)$.

The various derivations above allow to rewrite the whole set of governing equations and boundary equations of the problem as follows, by combining 
(5) and (14):

$$
\begin{aligned}
\forall \boldsymbol{v}^{\star}, \quad 2 \int_{\Omega} \eta \boldsymbol{D}\left(\boldsymbol{v}^{\star}\right): \boldsymbol{D}(\boldsymbol{v}) \mathrm{d} S-\int_{\Omega} p \operatorname{div} \boldsymbol{v}^{\star} \mathrm{d} S+ \\
\quad+\int_{\partial \Omega_{F}} \gamma \boldsymbol{t} \cdot\left(\operatorname{grad} \boldsymbol{v}^{\star}\right) \cdot \boldsymbol{t} \mathrm{d} l-\sum_{C_{i}} \epsilon \gamma \boldsymbol{v}^{\star} \cdot \boldsymbol{t}_{S} \cos \theta_{s}+ \\
\quad+\int_{\partial \Omega_{S}} \frac{1}{\beta} \boldsymbol{v}^{\star} .\left(\boldsymbol{v}-\boldsymbol{v}_{S}\right) \mathrm{d} l=0
\end{aligned}
$$

and

$$
\forall p^{\star}, \quad \int_{\Omega} p^{\star} \operatorname{div} \boldsymbol{v} \mathrm{d} S
$$

This variational formulation may also be arrived at by specializing to twodimensional flows the expression obtained by Ganesan and Tobiska [23] in a more general context of three-dimensional flows where the Laplace-Beltrami operator is used to avoid computing the curvature, but the way the contact angle is introduced is quite different. Here, the physical condition that applies along a contact line has been stated as (5), whereas the weak form must be written first in the procedure of [23], with an interpretation of the wetting force that is similar to Sikalo et al. [19].

\subsection{Numerical implementation}

A natural element method, which is a natural neighbour Galerkin method, is used to implement the above variational formulation. A NEM has already been applied to fluid dynamics by Martínez et al. [8] or González et al. [9], but based on the $\alpha$-NEM variant of Cueto et al. [7], which differs from the C-NEM variant of Yvonnet et al. [11] that we use here. The latter applies a visibility criterion to select natural neighbours in non convex fluid regions. Inclusion of surface tension in the C-NEM has been performed by Defauchy et al. [13] recently, but without avoiding the computation of curvature and without including fluid-solid contact.

The present implementation of the C-NEM uses the mixed formulation defined by (16) and (17), where three variables are introduced at each node, namely two velocity components and a pressure value. The nodes are scattered in the fluid domain and along its boundary, and a Voronoï cell results around each node. Integration over the fluid domain is performed by summing the integrals computed over all Voronoï cells. The velocity components 
are interpolated with the Sibson [24] continuous interpolant and integration is performed with the stabilized conforming nodal integration scheme defined by Chen et al. [25], whereas the pressure is taken constant over each Voronoï cell. This combination of different integration schemes has been shown by González et al. [26] not to satisfy strictly the Ladyshenskaya-Babuška-Brezzi [27, 28, 29] stability condition, but no spurious modes were found nevertheless by González et al. [9] in various practical applications to two-dimensional flows of incompressible fluids. The integration procedure is simpler along the boundary of the fluid domain, where velocities vary linearly between adjacent nodes.

The standard matrix structure of a mixed velocity-pressure formulation results from the discrete form of (16) and (17) in terms of the unknown nodal velocity and pressure noted as $\{\bar{V}, \bar{P}\}^{T}$ :

$$
\left[\begin{array}{cc}
A & C \\
C^{T} & 0
\end{array}\right]\left\{\begin{array}{l}
\bar{V} \\
\bar{P}
\end{array}\right\}=\left\{\begin{array}{l}
F \\
0
\end{array}\right\}
$$

where matrix $A$ is symmetric and vector $F$ includes the contributions of three terms pertaining to Laplace's law, wetting angle, and Navier slip. In the case of a shear-thinning fluid, matrix $A$ depends on $\bar{V}$ through the viscosity $\eta\left(\dot{\gamma}_{e q}\right)$, and an iterative procedure is performed at each time step in order to determine the viscosity at all nodes. A fixed-point method gives rapid convergence. If $b$ instead of $\beta$ is fixed in the definition of the Navier slip, the $F$ vector must also be updated at each of these iterations for a shearthinning fluid, since $\beta=b / \eta$. After completion of these procedures, the nodal positions and connections are updated from the velocities with a simple explicit scheme in this work, which implies that small time steps are used. Then, the computer programme, which uses MATLAB [30] for all numerical procedures, proceeds with finding the velocity and pressure fields at the next time step.

\section{Examples}

\subsection{Hollow cylinder contracted by surface tension}

As a first example, where an analytical solution can be used to validate the simulations, the model problem of the surface tension-driven contraction of a hollow cylinder is considered. The cylinder, made of an incompressible Newtonian fluid with viscosity $\eta_{0}$, has an unbounded length, so that velocities 


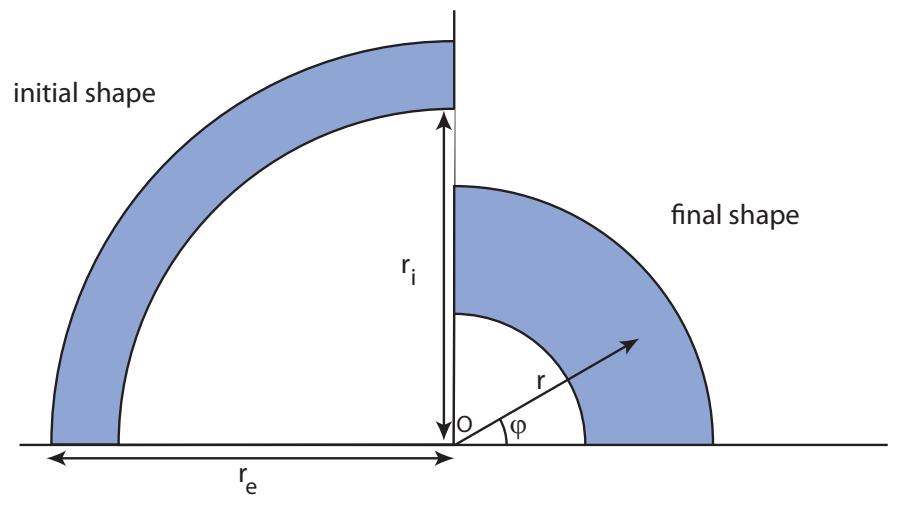

Figure 3: Geometry considered for the surface tension-driven contraction of a hollow cylinder. No pressure is applied on the inner and outer free surfaces.

are zero along its axis. No pressure is applied on the inner and outer surfaces, where the value of surface tension is $\gamma$. In these conditions, the hollow cylinder tends to shrink spontaneously until the central cavity closes. For given values of the inner and outer radii $r_{i}$ and $r_{e}$, the purely radial velocity field is easily obtained as

$$
v_{r}=-\frac{a}{r} \quad \text { with } \quad a=\frac{\gamma}{2 \eta_{0}}\left(\frac{1}{r_{i}}-\frac{1}{r_{e}}\right)^{-1} .
$$

Indeed, this ensures incompressiblity since $\partial v_{r} / \partial r+v_{r} / r=0$ and leads to stress components

$$
\sigma_{r r}=2 \eta_{0} \frac{a}{r^{2}}-p \quad \text { and } \quad \sigma_{\varphi \varphi}=-2 \eta_{0} \frac{a}{r^{2}}-p
$$

in cylindrical coordinates, with a uniform hydrostatic pressure

$$
p=\gamma\left(\frac{1}{r_{i}}-\frac{1}{r_{e}}\right)
$$

such that momentum balance $\partial \sigma_{r r} / \partial r+\left(\sigma_{r r}-\sigma_{\varphi \varphi}\right) / r=0$ is satisfied. Moreover, the boundary conditions given by Laplace's law are fulfilled, for $\sigma_{r r}$ takes the values $\gamma / r_{i}$ and $-\gamma / r_{e}$ on the inner and outer surfaces, respectively. The differential equation $\dot{r}_{i}=a / r_{i}$ governing the inner radius is integrated 
easily to obtain

$r_{i}(t)=-\frac{r_{e}(0)-r_{i}(0)}{2}\left(1+\frac{\gamma}{2 \eta_{0}} \frac{t}{r_{e}(0)-r_{i}(0)}-\frac{r_{e}(0)+r_{i}(0)}{r_{e}(0)-r_{i}(0)} \frac{1}{1+\frac{\gamma}{2 \eta_{0}} \frac{t}{r_{e}(0)-r_{i}(0)}}\right)$

and the corresponding history $r_{e}(t)=\sqrt{r_{i}^{2}(t)+r_{e}^{2}(0)-r_{i}^{2}(0)}$ of the outer radius results from the preservation of total fluid volume. It may be noted that complete closure of the inner cavity is obtained for

$$
t=\frac{2 \eta_{0}}{\gamma} \sqrt{r_{e}(0)-r_{i}(0)}\left(\sqrt{r_{e}(0)+r_{i}(0)}-\sqrt{r_{e}(0)-r_{i}(0)}\right) .
$$

For symmetry reasons, one quarter of the section of the cylinder is modeled, as shown in Fig. 3. An irregular set of 1210 nodes was distributed in the section and on its boundaries using the Gmsh free mesh generator developed by Geuzaine and Remacle [31], like for all other simulations in this paper. The initial inner and outer radii were $1 \mu \mathrm{m}$ and $1.2 \mu \mathrm{m}$, respectively, which were left to evolve during $0.09 \mathrm{~s}$ to give the final shape shown in Fig. 3 with radii of $0.388 \mu \mathrm{m}$ and $0.769 \mu \mathrm{m}$ for a viscosity of $10^{4} \mathrm{~Pa}$.s and a surface tension of $40 \mathrm{mN} / \mathrm{m}$. The stress components obtained through the section at the first time increment are shown in Fig. 4 and a good agreement is obtained with the above analytical solution, with some dispersion though. For instance, the standard deviation of the pressure field is 1.9 percent of the average value, but the latter is 0.01 percent close to the exact (uniform) pressure value. This dispersion is likely due to too simple interpolation and integration schemes, but it is acceptable for the applications considered and the use of more elaborate procedures, as proposed by González et al. [26], is left for future work. As shown in Fig. 5, the agreement is excellent when the evolutions of the inner and outer radii, or of the average pressure through the thickness, are considered. This confirms that the implementation of surface tension in the C-NEM is satisfactory and that the code is able to update the geometry correctly.

\subsection{Spread of a cylindrical droplet}

The above example involved surface tension only as a surface effect, and we consider now a flow where contact with a fixed rigid solid also is present. A cylindrical droplet of fluid with a semi-circular section of radius $R$ is laid on a solid substrate. If the wetting angle between the fluid and the solid 


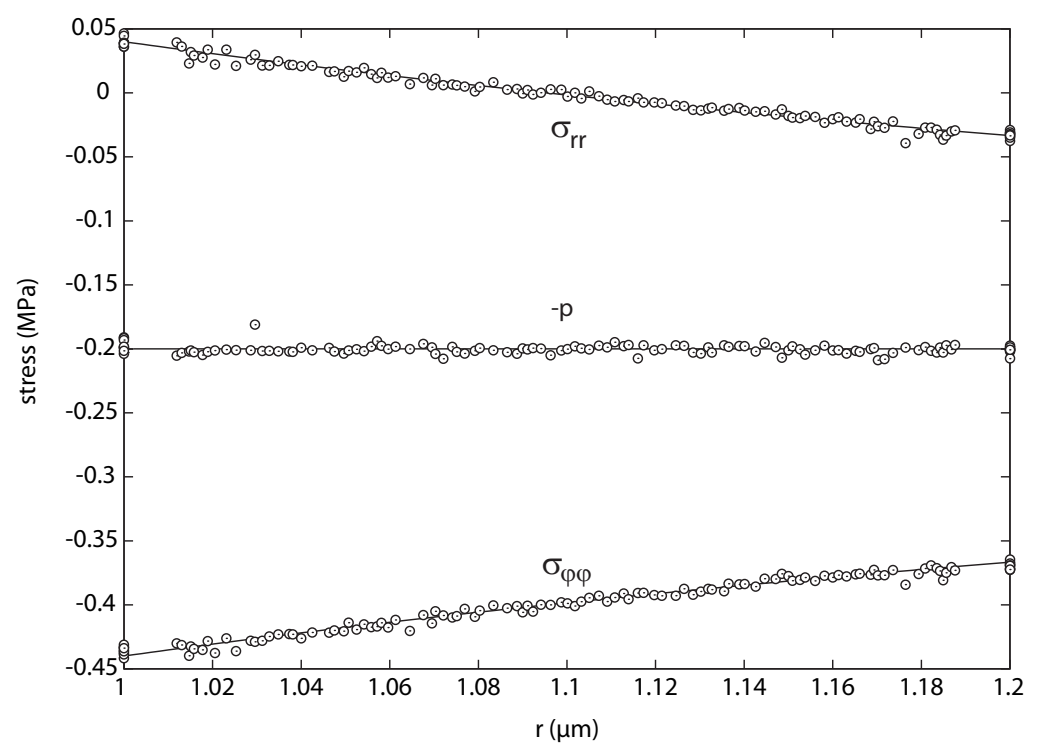

Figure 4: Hydrostatic pressure $(p)$, radial $\left(\sigma_{r r}\right)$ and hoop $\left(\sigma_{\varphi \varphi}\right)$ stresses through the thickness of the hollow cylinder considered when $r_{i}=1 \mu \mathrm{m}$ and $r_{e}=1.2 \mu \mathrm{m}$. Comparison between simulation (symbols) and analytical solution (solid lines). Only ten percent of nodes are shown for clarity.

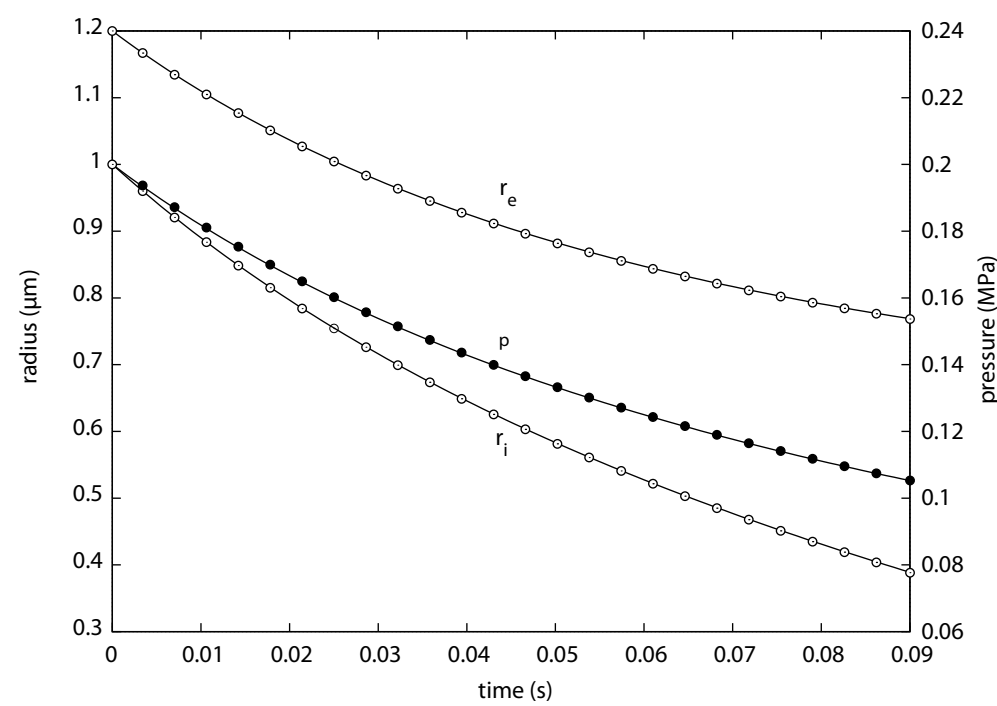

Figure 5: Evolution of the inner $\left(r_{i}\right)$ and outer $\left(r_{e}\right)$ radii of the cylinder, and of the average pressure through the thickness $(p)$. Comparison between simulation (symbols) and analytical solution (solid lines). Only ten percent of time steps are shown for clarity. 


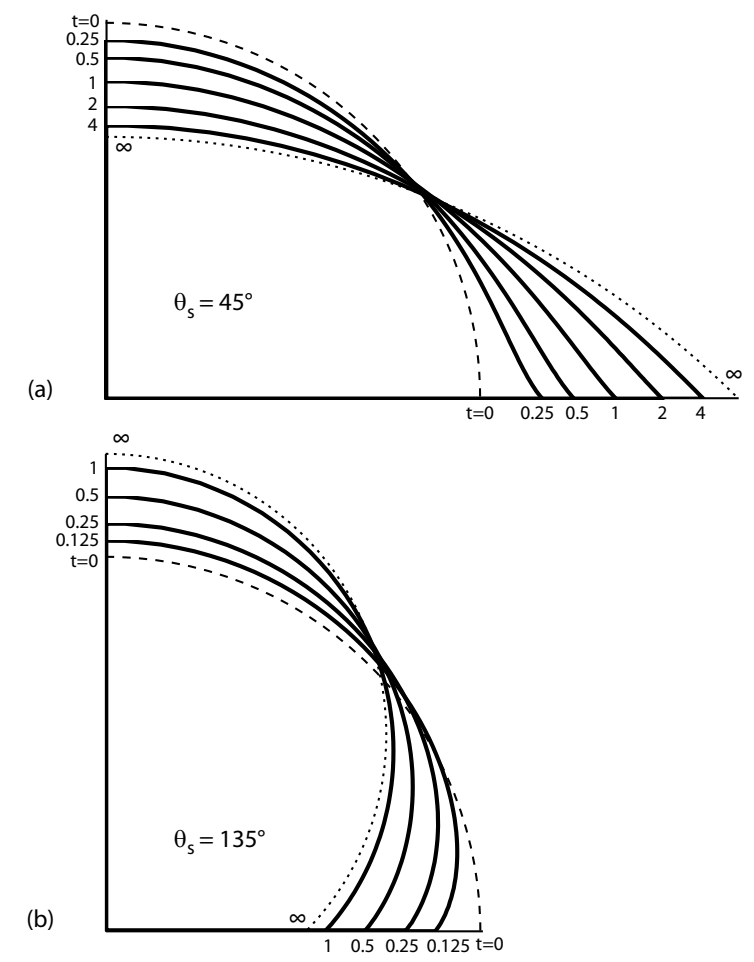

Figure 6: Initial (broken line, radius of $1 \mu \mathrm{m}$ ), final (dotted line) and intermediate (at times shown, in seconds) configurations taken by a cylindrical Newtonian drop that interacts with a substrate. Simulations used a viscosity of $10^{4}$ Pa.s, a surface tension of $40 \mathrm{mN} / \mathrm{m}$ and a Navier slip length of $50 \mathrm{~nm}$, with a wetting angle of either $45^{\circ}$ (a) or $135^{\circ}$ (b).

is equal to $90^{\circ}$, this is a stable configuration since gravity is neglected, and nothing happens. Otherwise, as illustrated in Fig. 6, the fluid flows until a new stable configuration is obtained, with the free surface in a circular arc of height $h$ and width $l$ such that

$$
l_{\infty}=2 R \sqrt{\frac{\pi}{2} \frac{1-\cos 2 \theta_{s}}{2 \theta_{s}-\sin 2 \theta_{s}}} \quad \text { and } \quad h_{\infty}=R \frac{1-\cos \theta_{s}}{\sin \theta_{s}} \sqrt{\frac{\pi}{2} \frac{1-\cos 2 \theta_{s}}{2 \theta_{s}-\sin 2 \theta_{s}}}
$$

as obtained easily from the preservation of fluid volume. For instance, $l_{\infty} / R=3.318$ and $h_{\infty} / R=0.687$ if $\theta_{s}=45^{\circ}$ (the drop spreads on a hydrophilic surface), or $l_{\infty} / R=1.049$ and $h_{\infty} / R=1.266$ if $\theta_{s}=135^{\circ}$ (the drop is repelled by a hydrophobic surface). These are exactly the limit values obtained by the simulations for these two wetting angles, where one half of the drop section was modeled for symmetry reasons, using 2592 nodes with refinement involving node distances of $10 \mathrm{~nm}$ near the fluid-solid interface. 


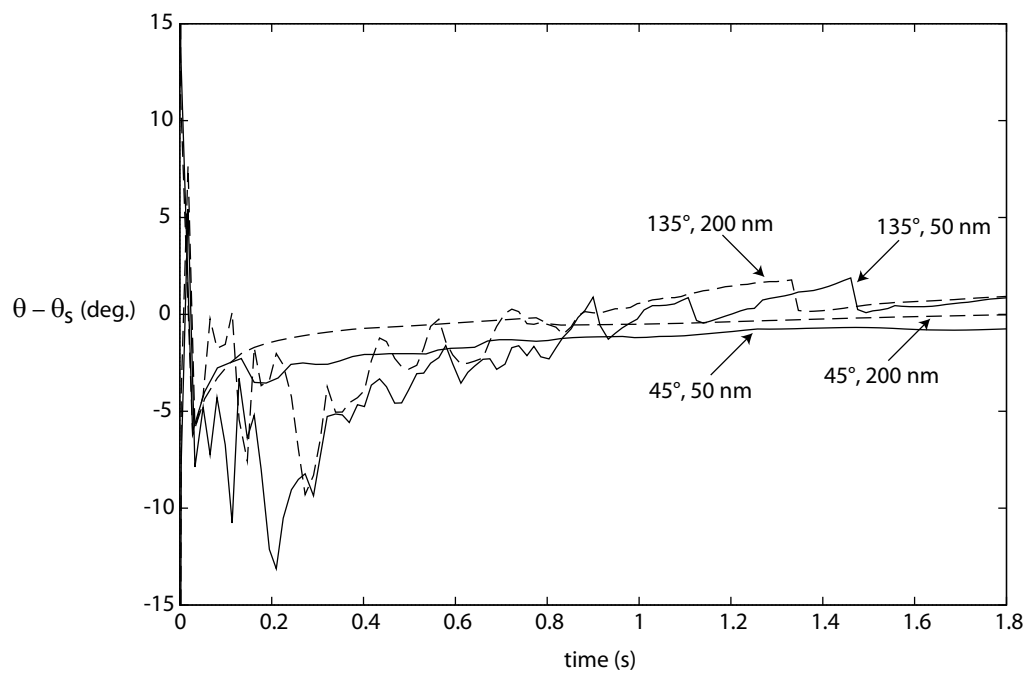

Figure 7: Difference between the contact angle and the static wetting angle obtained during the first stages of the spontaneous evolution of a Newtonian cylindrical droplet with a viscosity of $10^{4} \mathrm{~Pa} . \mathrm{s}$ and a surface tension of $40 \mathrm{mN} / \mathrm{m}$. Static wetting angles and Navier slip lengths as indicated.

The shapes shown in Fig. 6 are simpler than those obtained by Ganesan and Tobiska [23] for different wetting angles, but the simulations differ in at least two respects: the flow is two-dimensional, not axisymmetric, and there is no inertia here. As mentioned in the previous section, the contact angle results from the whole flow in the present simulations, where a dynamic wetting angle is not prescribed. The contact angle can be observed in Fig. 6 to evolve very quickly from its initial $90^{\circ}$ value to approach the static wetting angle. This is confirmed quantitatively in Fig. 7, where oscillations are due to the rapid node change at the contact point, like in [23], and where a faster and stabler evolution towards the limit value is observed for $\theta_{s}=45^{\circ}$ than for $\theta_{s}=135^{\circ}$. Moreover, the evolution of the contact angle towards its limit value is faster when the Navier slip length is increased from $50 \mathrm{~nm}$ to $200 \mathrm{~nm}$, i.e. when friction is reduced.

The evolution of the droplet height is shown in Fig. 8. The difference with the asymptotic value is found to decrease exponentially after a transient stage where the height changes very rapidly. This exponential decrease corresponds to a contact angle that is already close to its limit value and, consequently, to a very small net wetting force applied at the contact point. The characteristic time of the exponential decrease is found much shorter for $\theta_{s}=135^{\circ}$ than for 


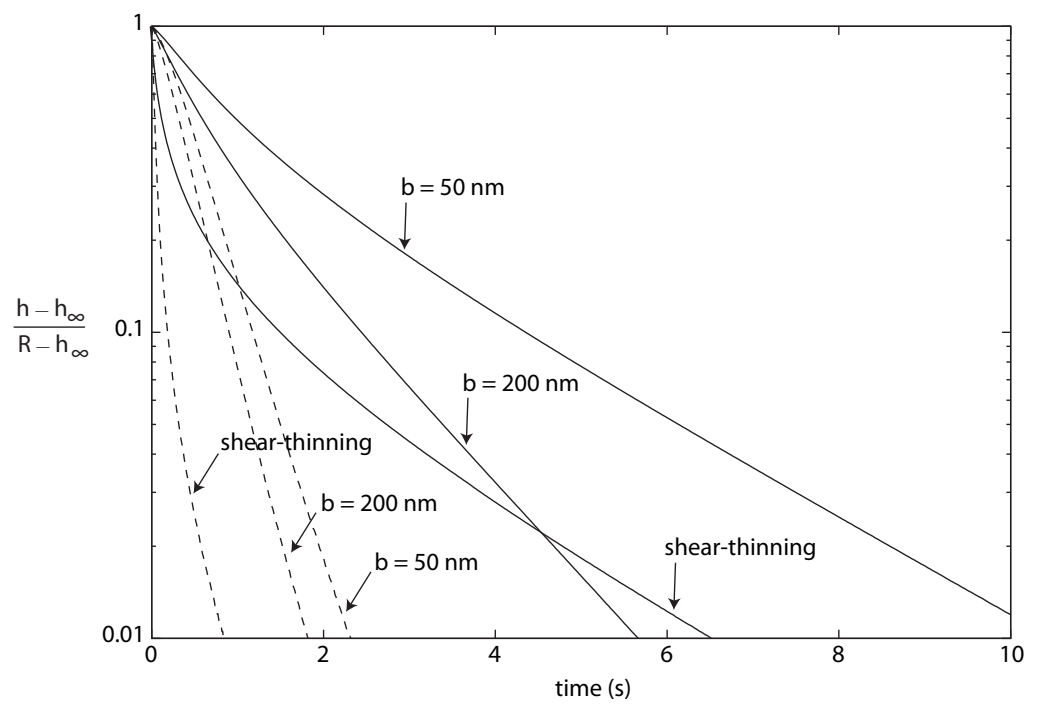

Figure 8: Evolution obtained for the normalized height change of a cylindrical droplet for a static wetting angle of either $45^{\circ}$ (solid lines) or $135^{\circ}$ (broken lines). Two values of the Navier slip length used, as indicated, with an initial radius $R$ of $1 \mu \mathrm{m}$ and a surface tension of $40 \mathrm{mN} / \mathrm{m}$. Same $\beta$ value used in the shear-thinning cases as for the Newtonian fluid with $b=50 \mathrm{~nm}$ and $\eta_{0}=10^{4}$ Pa.s.

$\theta_{s}=45^{\circ}$, which is consistent with the shape evolutions illustrated in Fig. 6 . Moreover, increasing the Navier slip length facilitates fluid flow and leads to shorter characteristic times (Fig. 8). Our rheometric measurements on a polystyrene melt of $35 \mathrm{~kg} / \mathrm{mol}$ have demonstrated a Newtonian plateau with $\eta_{0}=10^{4} \mathrm{~Pa} . \mathrm{s}$ at $110^{\circ} \mathrm{C}$ followed by a shear thinning behaviour that could be fitted well with a Carreau-Yasuda law

$$
\eta=\eta_{0}\left[1+\left(\frac{\dot{\gamma}_{e q}}{\dot{\gamma}_{0}}\right)^{a}\right]^{\frac{n-1}{a}}
$$

using $\dot{\gamma}_{0}=0.075 / \mathrm{s}, a=0.93$ and $n=0.49$. Taking shear-thinning into account in the simulations, but keeping the surface tension and slip coefficient constant, leads to a much faster evolution of the droplet shape, as can be noted in Fig. 8. At the very beginning, the net wetting force at the contact point is large enough to induce high strain rates associated with low viscosities and the flow is very fast, but this force weakens when the contact angle gets close to the static wetting angle, the flow slows down, the viscosity increases towards its Newtonian limit value, and the previous exponential decay is recovered. 


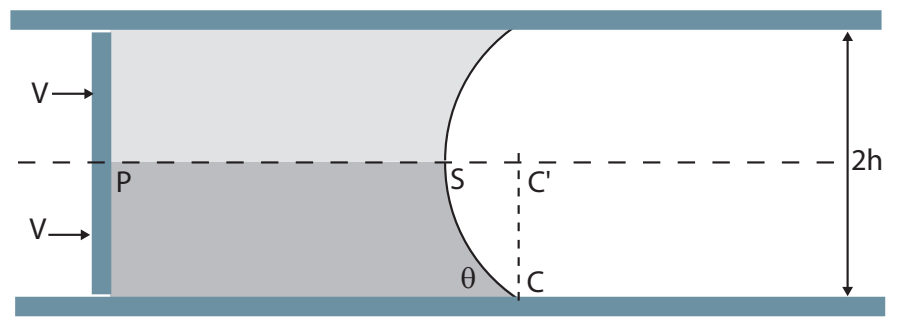

Figure 9: The piston-driven problem considered, where the steady shape of a meniscus between two plates is studied. For symmetry reasons, the lower part of the fluid region only, shown in dark grey, is modeled.

\subsection{Steady translation of a meniscus between two plates}

The steady shape of a fluid meniscus that translates in a capillary tube has been investigated experimentally by Hoffman [32], whose results have stimulated many theoretical studies initiated by the pionneering finite element iterative procedure of Lowndes [33]. In the present context of twodimensional flows, the geometry studied is not axisymmetric but a flow between two parallel plates is considered instead, like in the dissipative particle dynamics simulations of Cupelli et al. [34]. This is also the case in the work of Deganello et al. [18], who applied the level-set method with no-slip at the fluid-solid interface. As shown in Fig. 9, the distance between the fixed plates is $2 h$ and the piston moves with velocity $V$. For symmetry reasons, the lower half of the figure is modeled only. The initial shape of the free surface is a circular arc defined by the static wetting angle, i.e. it corresponds to the static equilibrium. Consequently, the initial value of distance $S C^{\prime}$ is $h\left(1-\sin \theta_{s}\right) / \cos \theta_{s}$. The piston is then pushed until the shape of the free surface stabilizes, which can be characterized by the necessary (and sufficient, in practice) condition that distance $S C^{\prime}$ becomes constant. Various lengths $P S$ of the initial fluid domain have been tested, from $3 h$ to $6 h$ without significant effect, and therefore a length of $P S=3 h$ is used, which can be compared with the values of $2 h$ used in [33] and about 2.5h used [35] and [36].

It is recalled that the approach used here prescribes the net wetting force (5) at the contact line, which differs from previous studies where the angle at which the free surface meets the solid boundary was imposed instead. The latter prescription may lead to specific numerical difficulties, as discussed in detail by Sprittles and Shikhmurzaev [36], and induces an additional term in the numerical formulation of Bazhlekov and Chesters [35], for instance, that is similar to the first term in (5). Here, in agreement with the experimental 

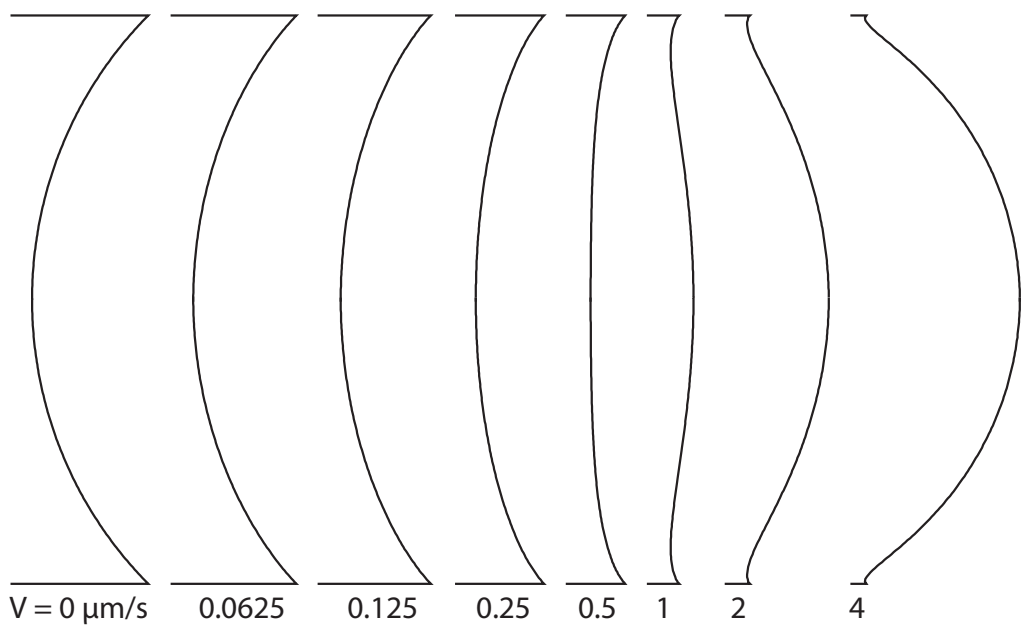

Figure 10: Steady meniscus shapes obtained for a Newtonian fluid with various piston velocities, for a distance between fixed plates of $2 \mu \mathrm{m}$, a viscosity of $10^{4} \mathrm{~Pa} . \mathrm{s}$, a surface tension of $40 \mathrm{mN} / \mathrm{m}$, a static wetting angle of $45^{\circ}$ and a Navier slip length of $50 \mathrm{~nm}$.

observations of Blake et al. [37] in the context of curtain coating, the contact angle $\theta$ stabilizes at a value that is a function not solely of material properties and piston speed but also of the whole flow field.

A half-distance between the plates of $l=1 \mu \mathrm{m}$ was used in the simulations, and 2185 nodes were defined to model the fluid region, with refinement involving node distances of $10 \mathrm{~nm}$ in the vicinity of the free surface and of the fluid-solid interface. The same material constants are used as in the previous examples. Piston velocities $V$ between $0.0625 \mu \mathrm{m} / \mathrm{s}$ and $4 \mu \mathrm{m} / \mathrm{s}$ were considered, which lead to a capillary number $C_{a}=\eta_{0} V / \gamma$ between 0.016 and 1 . Frictionless slip was prescribed on the piston-fluid interface, and the Navierslip condition was applied at the plate-fluid interface. A value $\theta_{s}=45^{\circ}$ was chosen for the static wetting angle. Fig. 10 illustrates (with symmetry applied) the steady meniscus profiles obtained, with a manifest effect of the piston velocity, which reverses the meniscus curvature from concave to convex. Because of the rolling process that takes place at the contact point in the steady state, with this point being defined by always changing nodes, it has not been possible to evaluate the steady dynamic contact angle $\theta$ precisely, and the apparent contact angle $\theta_{a}$ has been computed instead. This angle is consistent with the measurement procedure of Hoffman [32] and can 
be deduced from the steady $S C^{\prime}$ distance as

$$
\theta_{a}=\cos ^{-1}\left(\frac{2 h S C^{\prime}}{h^{2}+S C^{\prime 2}}\right)
$$

Fig. 11 shows the effect of the capillary number on the apparent contact angle. An agreement with the Hoffman-Voinov-Tanner law [32, 38, 39], that predicts a linear relationship with the variables used in the figure, is observed for small capillary numbers. The subsequent downwards bend of the curves is consistent with a limit contact angle of $180^{\circ}$.

Fig. 11 also shows the influence of the Navier slip length $b=\beta \eta_{0}$ for a Newtonian fluid, which is significant even for low capillary numbers. An increase of the slip length induces a reduction of the apparent contact angle, which is consistent with the contact angle being equal to the static wetting angle and independent of the capillary number when the fluid-interface is frictionless. The fluid plug merely translates as a rigid body in these conditions and the initial static equilibrated shape is preserved. The problem considered can be characterized completely by two non-dimendional numbers, namely the capillary number $C_{a}$ and the ratio $b / h$ of the slip length to channel (half-)height, and consequently the above trend can also be interpreted in terms of a fixed slip length and a decreasing channel height. Thus, the influence of the capillary number on the meniscus shape is affected significantly when the length scale of the problem becomes of the same order as a characteristic material length, which is a typical size effect at micro or nano scale.

When a shear-thinning behaviour is taken into account, the high strain rates that are involved in the vicinity of the contact line reduce the viscosity drastically and, consequently, the contact angle is more easily adjusted towards the static value. For a slip coefficient $\beta=50 \times 10^{-13} \mathrm{~m} / \mathrm{s} / \mathrm{Pa}$, a comparison is given in Fig. 11 between the shear-thinning behaviour (solid circles) given by (25) and the limit case of a Newtonian fluid (open circles) with the same $\beta$ value, using $\eta_{0}=10^{4} \mathrm{~Pa}$.s. Note that the latter viscosity is used to define the same capillary number in both cases in Fig. 11.

\section{Conclusions}

The natural element method could be used successfully to simulate twodimensional viscous flows where interfacial effects must be taken into account, at zero Reynolds numbers, for applications at the micro and nano scales. The 


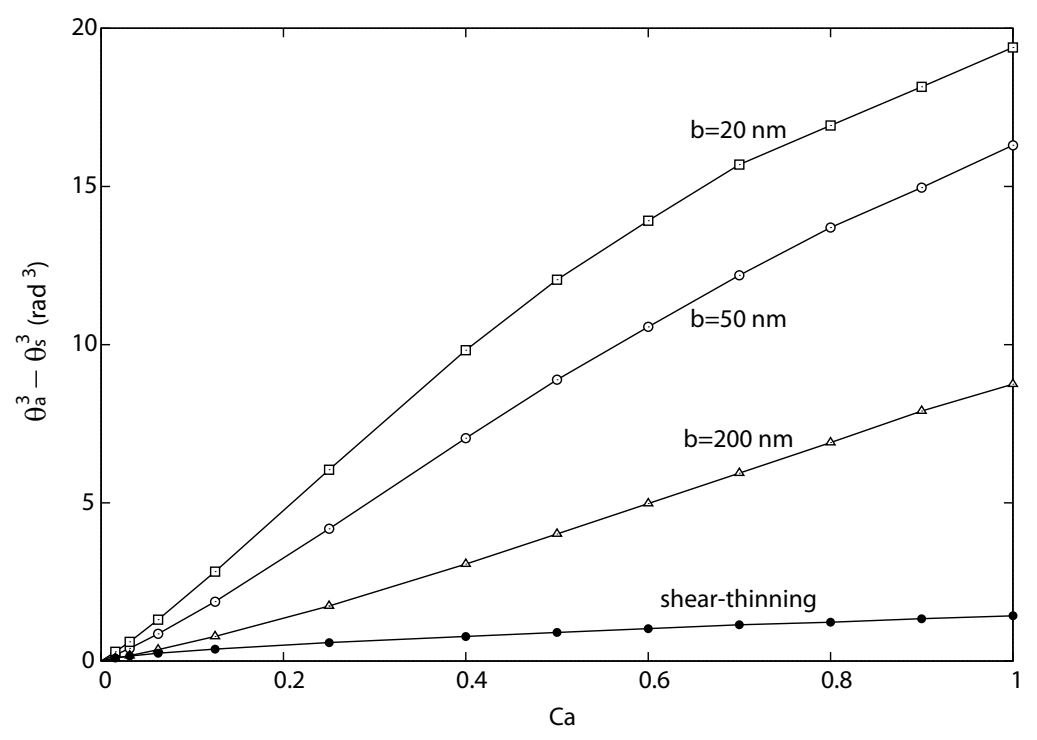

Figure 11: Effect of the capillary number $C_{a}$ on $\theta_{a}^{3}-\theta_{s}^{3}$, where $\theta_{a}$ is the meniscus apparent contact angle and $\theta_{s}$ the static wetting angle (both angles in radians), for a Newtonian fluid with various Navier slip lengths $b$. The effect of changing from a Newtonian fluid with $b=50 \mathrm{~nm}$ to a shear-thinning fluid with the same slip coefficient is also shown. A wetting angle $\theta=45^{\circ}$ and a $2 \mu \mathrm{m}$ distance between plates were used.

variational formulation used incorporates surface tension on the free surfaces, and a net wetting force is applied at the contact line where the fluid reaches a solid surface. Moreover, the Navier-slip condition is applied along the fluidsolid interface. No dynamic wetting angle is prescribed, and the contact angle obtained results from the other material parameters and from overall flow conditions.

This work extends previous applications of the natural element method to fluids, where surface tension was either ignored or included in a less suitable manner, and where fluid-solid contact was absent. A comparison with an analytical solution in a simple surface tension-driven flow has been given, and fluid-solid contact has been discussed in a transient case and in a steady problem. The effect of the slip contact length has been analyzed, as well as the influence of shear-thinning. 


\section{Acknowledgements}

The authors are grateful to L. Illoul for making his C-NEM routines available. Support from the Agence Nationale de la Recherche through project SINCRONE is acknowledged.

\section{References}

[1] S.Y. Chou, P.K. Krauss, P.J. Renstrom, Imprint lithography with 25nanometer resolution, Science 272 (1996) 85-87

[2] E. Lauga, M.P. Brenner, H.A. Stone, Microfluidics: the no-slip boundary condition, in: J. Foss, C. Tropea and A. Yarin (Eds.), Handbook of Experimental Fluid Mechanics, Springer, 2005, pp. 1219-1240.

[3] C. Neto, D.R. Evans, E. Bonaccurso, H.-J. Butt, V.S.J. Craig, Boundary slip in Newtonian liquids: a review of experimental studies, Rep. Prog. Phys. 68 (2005) 2859-2897.

[4] L. Traversoni, Natural neighbour finite elements, in: W.R. Blain, K.L. Katsifarakis (Eds.), Hydraulic Engineering Software V, vol. 2, Computational Mechanics Publications, Southampton, 1994, pp. 291-297.

[5] J. Braun, M. Sambridge, A numerical method for solving partial differential equations on highly irregular evolving grids, Nature 376 (1995) 655-660.

[6] N. Sukumar, B. Moran, T. Belytschko, The natural element method in solid mechanics, Int. J. Numer. Meth. Engng 43 (1998) 839-887.

[7] E. Cueto, M. Doblaré, L. Gracia, Imposing essential boundary conditions in the natural element method by means of density-scaled $\alpha$ shapes, Int. J. Numer. Meth. Engng 49 (2000) 519-546.

[8] M.A. Martínez, E. Cueto, M. Doblaré, F. Chinesta, Natural element meshless simulation of flows involving short fiber suspensions, J. NonNewtonian Fluid Mech. 115 (2003) 51-78.

[9] D. González, E. Cueto, F. Chinesta, M. Doblaré, A natural element updated Lagrangian strategy for free-surface fluid dynamics, J. Comput. Phys. 223 (2007) 127-150. 
[10] R.Ata, A. Soulaïmani, F. Chinesta, The natural volume method (NVM): Presentation and application to shallow water inviscid flows, Int. J. Numer. Meth. Fluids 59 (2009) 19-45.

[11] J. Yvonnet, D. Ryckelynck, P. Lorong, F. Chinesta, A new extension of the natural element method for non-convex and discontinuous problems: the constrained natural element method (C-NEM), Int. J. Numer. Meth. Engng 60 (2004), 1451-1474.

[12] M. Darbani, A. Ouahsine, P. Villon, H. Naceur, H. Smaoui, Meshless method for shallow water equations with free surface flow, Appl. Math. Comput. 217 (2011) 5113-5124.

[13] D. Defauchy, G. Régnier, I. Amran, P. Peyre, A. Ammar, F. Chinesta, Towards a numerical simulation of direct manufacturing of thermoplastic parts by powder laser sintering, in: E. Oñate, D.R.J. Owen, P. Peric, B. Suárez (Eds.), Computational Plasticity XI. Fundamentals and Applications, CIMNE Publications, Barcelona, 2011, pp. 688-699.

[14] E. Cueto, N. Sukumar, B. Calvo, M.A. Martínez, J. Cegoñino, M. Doblaré, Overview and recent advances in natural neighbour Galerkin methods, Arch. Comp. Meth. Engng. 10 (2003) 307-384.

[15] G. Karniadakis, A. Beskok, N. Aluru, Microflows and Nanoflows. Fundamentals and Solutions, Springer, 2005.

[16] P.-G. de Gennes, F. Brochard-Wyart, D. Quéré, Capillarity and Wetting Phenomena: Drops, Bubbles, Pearls, Waves, Springer Verlag, 2002.

[17] Y.D. Shikhmurzaev, Capillary Flows with Forming Interfaces, Chapman \& Hall/CRC, Boca Raton FL, 2008.

[18] D. Deganello, T.N. Croft, A.J. Williams, A.S. Lubansky, D.T. Gethin, T.C. Claypole, Numerical simulation of dynamic contact angle using a force based formulation, J. Non-Newtonian Fluid Mech. 166 (2011) 900-907.

[19] S̆. Škalo, H.-D. Wilhelm, I.V. Roisman, S. Jakirlić, C. Tropea, Dynamic contact angle of spreading droplets: Experiments and simulations, Phys. Fluids 17 (2005) 062103-1-13. 
[20] C. Huh, L.E. Scriven, Hydrodynamic model of steady movement of a solid/liquid fluid contact line, J. Colloid Interface Sci. 35 (1971) 85-101.

[21] L.M. Hocking, A moving interface. Part 2: The removal of the force singularity by a slip flow, J. Fluid Mech. 79 (1977) 209-229.

[22] K.J. Ruschak, A method for incorporating free boundaries with surface tension in finite element fluid-flow simulators, Int. J. Numer. Meth. Engng 15 (1980) 639-648.

[23] S. Ganesan, L. Tobiska, Modelling and simulation of moving contact line problems with wetting effects, Comput. Visual Sci. 12 (2009) 329-336.

[24] R. Sibson, A vector identity for the Dirichlet tesselation, Math. Proc. Camb. Phil. Soc. 87 (1980) 151-155.

[25] J.-S. Chen, C.-T. Wu, S. Yoon, Y. You, A stabilized conforming nodal integration for Galerkin mesh-free method, Int. J. Numer. Meth. Engng 50 (2001) 435-466.

[26] D. González, E. Cueto, M. Doblaré, Volumetric locking in natural neighbour Galerkin methods, Int. J. Numer. Meth. Engng 61 (2004) 611-632.

[27] O.A. Ladyzhenskaya, The Mathematical Theory of Viscous Incompressible Flow, second ed., Gordon and Breach, New York, 1969.

[28] I. Babuška, The finite element method with Lagrangian multipliers, Numer. Math. 20 (1973) 179-192.

[29] F. Brezzi, On the existence, uniqueness and approximation of saddlepoint problems arising from Lagrange multipliers, RAIRO 8 (1974) 129151.

[30] MATLAB R2011a, The MathWorks Inc., Natick MA, USA, 2011.

[31] C. Geuzaine, J.-F. Remacle, Gmsh: a three-dimensional finite element mesh generator with built-in pre- and post-processing facilities, Int. J. Numer. Meth. Engng 79 (2009) 1309-1331.

[32] R.L. Hoffman, A study of the advancing interface- I. Interface shape in liquid-gas systems, J. Colloid Interface Sci. 50 (1975) 228-241. 
[33] J. Lowndes, The numerical simulation of the steady movement of a fluid meniscus in a capillary tube, J. Fluid Mech. 101 (1980) 631-646.

[34] C. Cupelli, B. Henrich, T. Glatzel, R. Zengerle, M. Moseler, M. Santer, Dynamic capillary wetting studied with dissipative particle dynamics, New J. Phys.10 (2008) 043009.

[35] I.B. Bazhlekov, A.K. Chesters, Numerical investigation of the dynamic influence of the contact line region on the macroscopic meniscus shape, J. Fluid Mech. 329 (1996) 137-146.

[36] J.E. Sprittles, Y.D. Shikhmurzaev, Finite element framework for describing dynamic wetting phenomena, Int. J. Numer. Meth. Fluids 68 (2012) 1257-1298.

[37] T.D. Blake, M. Bracke, Y.D. Shikhmurzaev, Experimental evidence of nonlocal hydrodynamic influence on the dynamic contact angle, Phys. Fluids 11 (1999) 1995-2007.

[38] O.V. Voinov, Hydrodynamics of wetting, Fluid Dyn. 11 (1976) 714-721.

[39] L.H. Tanner, The spreading of silicone oil drops on horizontal surfaces, J. Phys. D 12 (1979) 1473-1484. 